\title{
Fast BF-ICrA Method for the Evaluation of MO-ACO Algorithm for WSN Layout
}

\author{
Jean Dezert \\ ONERA - DTIS \\ The French Aerospace Lab \\ Palaiseau, France. \\ jean.dezert@onera.fr
}

\author{
Stefka Fidanova \\ Inst. of I\&C Tech. \\ Bulgarian Academy of Sciences \\ Sofia, Bulgaria. \\ stefka@parallel.bas.bg
}

\author{
Albena Tchamova \\ Inst. of I\&C Tech. \\ Bulgarian Academy of Sciences \\ Sofia, Bulgaria. \\ tchamova@bas.bg
}

\begin{abstract}
In this paper, we present a fast Belief Function based Inter-Criteria Analysis (BF-ICrA) method based on the canonical decomposition of basic belief assignments defined on a dichotomous frame of discernment. This new method is then applied for evaluating the Multiple-Objective Ant Colony Optimization (MO-ACO) algorithm for Wireless Sensor Networks (WSN) deployment.
\end{abstract}

Keywords: Inter-Criteria Analysis, belief functions, information fusion, canonical decomposition, PCR5 rule.

\section{INTRODUCTION}

$\mathbf{I}$ $\mathrm{N}$ OUR previous work [1] we propose a new and improved version of classical Atanassov's InterCriteria Analysis (ICrA) [2] - [4] approach based on Belief Functions (BFICrA). This method proposes a better construction of InterCriteria Matrix that fully exploits all the information of the score matrix, and the closeness measure of agreement between criteria based on belief interval distance. In [5], we show how the fusion of many sources of evidences represented by Basic Belief Assignments (BBAs) defined on a same dichotomous frame of discernment can be fast and easily done thanks to the Proportional Conflict Redistribution rule no.5 based canonica decomposition of the BBAs, proposed recently in [6]. In the recent paper we consider $\mathrm{BF}-\mathrm{ICrA}$ based on this promising technique. Then we show how to apply it for the evaluation of the Multiple-Objective Ant Colony Optimization (MO-ACO) algorithm for Wireless Sensor Networks (WSN) deployment. After a condensed presentation of basics of belief functions in Section II, including the short description of canonical decomposition of dichotomous BBAs approach, and the main steps of fast fusion method of dichotomous BBAs, in Section III the BF-ICrA method is described and analyzed. Section IV is devoted to the multi-objective ACO algorithm. In Section $\mathrm{V}$ the results of the fast BF-ICrA method with the MOACO algorithm for WSN layout deployment is presented and discussed. Conclusion is given in Section VI.

\section{BASICS OF BELIEF FUNCTIONS}

\section{A. Basic definitions}

Belief functions (BF) have been introduced by Shafer in [7] to model epistemic uncertainty and to combine distinct sources of evidence thanks to Dempster's rule of combination.
In Shafer's framework, we assume that the answer ${ }^{1}$ of the problem under concern belongs to a known finite discrete frame of discernment (FoD) $\Theta=\left\{\theta_{1}, \theta_{2}, \ldots, \theta_{n}\right\}$, with $n>1$, and where all elements of $\Theta$ are mutually exclusive and exhaustive. The set of all subsets of $\Theta$ (including empty set $\emptyset$ and $\Theta$ ) is the power-set of $\Theta$ denoted by $2^{\Theta}$. A proper Basic Belief Assignment (BBA) associated with a given source of evidence is defined [7] as a mapping $m(\cdot): 2^{\Theta} \rightarrow[0,1]$ satisfying $m(\emptyset)=0$ and $\sum_{A \in 2^{\Theta}} m(A)=1$. The quantity $m(A)$ is called the mass of $A$ committed by the source of evidence. Belief and plausibility functions are respectively defined from a proper BBA $m(\cdot)$ by

$$
\operatorname{Bel}(A)=\sum_{B \in 2^{\Theta} \mid B \subseteq A} m(B)
$$

and

$$
P l(A)=\sum_{B \in 2^{\Theta} \mid A \cap B \neq \emptyset} m(B)=1-\operatorname{Bel}(\bar{A}) .
$$

where $\bar{A}$ is the complement of $A$ in $\Theta$.

$\operatorname{Bel}(A)$ and $\operatorname{Pl}(A)$ are usually interpreted respectively as lower and upper bounds of an unknown (subjective) probability measure $P(A)$. The quantities $m(\cdot)$ and $\operatorname{Bel}(\cdot)$ are oneto-one and linked by the Möbius inverse formula (see [7], p. 39). $A$ is called a Focal Element (FE) of $m(\cdot)$ if $m(A)>0$. When all focal elements are singletons, $m(\cdot)$ is called a Bayesian BBA [7] and its corresponding $\operatorname{Bel}(\cdot)$ function is equal to $P l(\cdot)$ and they are homogeneous to a (subjective) probability measure $P(\cdot)$. The vacuous BBA, representing a totally ignorant source, is defined as $m_{v}(\Theta)=1$. A dichotomous BBA is a BBA defined on a FoD which has only two proper subsets, for instance $\Theta=\{A, \bar{A}\}$ with $A \neq \Theta$ and $A \neq \emptyset$. A dogmatic BBA is a BBA such that $m(\Theta)=0$. If $m(\Theta)>0$ the BBA $m(\cdot)$ is nondogmatic. A simple BBA is a BBA that has at most two focal sets and one of them is $\Theta$. A dichotomous non dogmatic mass of belief is a BBA having three focal elements $A, \bar{A}$ and $A \cup \bar{A}$ with $A$ and $\bar{A}$ subsets of $\Theta$.

In his Mathematical Theory of Evidence [7], Shafer proposed to combine $s \geq 2$ distinct sources of evidence repre-

\footnotetext{
${ }^{1}$ i.e. the solution, or the decision to take
} 
sented by BBAs with Dempster's rule (i.e. the normalized conjunctive rule), which unfortunately behaves counterintuitively both in high and low conflicting situations as reported in [8][11]. In our previous works (see [12], Vol. 2 and Vol. 3 for full justification and examples) we did propose new rules of combination based on different Proportional Conflict Redistribution (PCR) principles, and we have shown the interest of the PCR rule No 5 (PCR5) for combining two BBAs, and PCR rule No 6 (PCR6) for combining more than two BBAs altogether [12], Vol. 2. PCR6 coincides with PCR5 when one combines two sources. The difference between PCR5 and PCR6 lies in the way the proportional conflict redistribution is done as soon as three (or more) sources are involved in the fusion. PCR5 transfers the conflicting mass only to the elements involved in the conflict and proportionally to their individual masses, so that the specificity of the information is entirely preserved in this fusion process.

The general (complicate) formulas for PCR5 and PCR6 rules are given in [12], Vol. 2. The fusion of two BBAs based on PCR5 (or PCR6) rule which will be use for canonical decomposition of a dichotomous BBA is obtained by the formula

$$
\begin{gathered}
m_{P C R 5}(X)=\sum_{\substack{X_{1}, X_{2} \in 2^{\Theta} \\
X_{1} \cap X_{2}=X}} m_{1}\left(X_{1}\right) m_{2}\left(X_{2}\right)+ \\
\sum_{\substack{X_{2} \in 2^{\Theta} \\
X_{2} \cap X=\emptyset}}\left[\frac{m_{1}(X)^{2} m_{2}\left(X_{2}\right)}{m_{1}(X)+m_{2}\left(X_{2}\right)}+\frac{m_{2}(X)^{2} m_{1}\left(X_{2}\right)}{m_{2}(X)+m_{1}\left(X_{2}\right)}\right]
\end{gathered}
$$

where all denominators in (3) are different from zero. If a denominator is zero, that fraction is discarded.

From the implementation point of view, PCR6 is simpler to implement than PCR5. For convenience, very basic (not optimized) Matlab ${ }^{\mathrm{TM}}$ codes of PCR5 and PCR6 fusion rules can be found in [12], [13] and from the toolboxes repository on the web [14]. The main drawback of PCR5 and PCR6 rules is their very high combinatorial complexity when the number of source is big, as well as the cardinality of the FoD. In this case, PCR5 or PCR6 rules cannot be used directly because of memory overflow. Even for combining BBAs defined on a simple dichotomous FoD as those involved in the Inter-Criteria Analysis (ICrA), the computational time for combining more than 10 sources can take several hours ${ }^{2}$. That is why a fast fusion method to combine dichotomous BBAs is necessary, and we present it in the next subsections.

\section{B. Canonical decomposition of dichotomous $B B A$}

A FoD $\Theta=\{A, \bar{A}\}$ is called dichotomous if it consists of only two proper subsets $A$ and $\bar{A}$ with $A \cup \bar{A}=\Theta$ and $A \cap \bar{A}=\emptyset$, where $\bar{A}$ is the complement of $A$ in $\Theta$ and $A$ is different from $\Theta$ and from Empty-Set. We consider a given proper BBA $m(\cdot): 2^{\Theta} \rightarrow[0,1]$ of the general form

$$
m(A)=a, \quad m(\bar{A})=b, \quad m(A \cup \bar{A})=1-a-b
$$

${ }^{2}$ with a MacBook Pro $2.8 \mathrm{GHz}$ Intel Core i7 with 16 Go $1600 \mathrm{MHz}$ DDR3 memory running Matlab ${ }^{\mathrm{TM}} \mathrm{R} 2018 \mathrm{a}$.
The canonical decomposition problem consists in finding the two following simple proper BBAs $m_{p}$ and $m_{c}$ of the form

$$
\begin{aligned}
& m_{p}(A)=x, \quad m_{p}(A \cup \bar{A})=1-x \\
& m_{c}(\bar{A})=y, \quad m_{c}(A \cup \bar{A})=1-y
\end{aligned}
$$

with $(x, y) \in[0,1] \times[0,1]$, such that $m=$ Fusion $\left(m_{p}, m_{c}\right)$, for a chosen rule of combination denoted by Fusion $(\cdot, \cdot)$. The simple BBA $m_{p}(\cdot)$ is called the pro- $B B A$ (or pro-evidence) of $A$, and the simple BBA $m_{c}(\cdot)$ the contra-BBA (or contraevidence) of $A$. The BBA $m_{p}(\cdot)$ is interpreted as a source of evidence providing an uncertain evidence in favor of $A$, whereas $m_{c}(\cdot)$ is interpreted as a source of evidence providing an uncertain contrary evidence about $A$.

In [6], we have shown that this decomposition is possible with Dempster's rule only if $0<a<1,0<b<1$ and $a+b<1$, and we have $x=\frac{a}{1-b}$ and $y=\frac{b}{1-a}$. However, any dogmatic BBA $m(A)=a, m(\bar{A})=b$ with $a+b=1$ is not decomposable from Dempster's rule for the case when $(a, b) \neq(1,0)$ and $(a, b) \neq(0,1)$, and the dogmatic BBAs $m(A)=1, m(\bar{A})=0$, or $m(A)=0, m(\bar{A})=1$ have infinitely many decompositions based on Dempster's rule of combination. We have also proved that this canonical decomposition cannot be done from conjunctive, disjunctive, Yager's [15] or Dubois-Prade [16] rules of combination, neither from the averaging rule. The main result of [6] is that this canonical decomposition is unique and is always possible in all cases using the PCR5 rule of combination. This is very useful to implement a fast efficient approximating fusion method of dichotomous BBAs as presented in details in [5]. We recall the following two important theorems proved in [6].

Theorem 1: Consider a dichotomous FoD $\Theta=\{A, \bar{A}\}$ with $A \neq \Theta$ and $A \neq \emptyset$ and a nondogmatic BBA $m(\cdot): 2^{\Theta} \rightarrow[0,1]$ defined on $\Theta$ by $m(A)=a, m(\bar{A})=b$, and $m(A \cup \bar{A})=$ $1-a-b$, where $a, b \in[0,1]$ and $a+b<1$. Then the BBA $m(\cdot)$ has a unique canonical decomposition using PCR5 rule of combination of the form $m=\operatorname{PCR} 5\left(m_{p}, m_{c}\right)$ with proevidence $m_{p}(A)=x, m_{p}(A \cup \bar{A})=1-x$ and contra-evidence $m_{c}(\bar{A})=y, m_{c}(A \cup \bar{A})=1-y$ where $x, y \in[0,1]$.

Theorem 2: Any dogmatic BBA defined by $m(A)=a$ and $m(\bar{A})=b$, where $a, b \in[0,1]$ and $a+b=1$, has a canonical decomposition using PCR5 rule of combination of the form $m=P C R 5\left(m_{p}, m_{c}\right)$ with $m_{p}(A)=x, m_{p}(A \cup \bar{A})=1-x$ and $m_{c}(\bar{A})=y, m_{c}(A \cup \bar{A})=1-y$ where $x, y \in[0,1]$.

Theorems $1 \& 2$ prove that the decomposition based on PCR5 always exists and it is unique for any dichotomous (nondogmatic, or dogmatic) BBA.

For the case of dichotomous nondogmatic BBA considered in Theorem 1, one has to find $x$ and $y$ solutions of the system

$$
\begin{aligned}
& a=x(1-y)+\frac{x^{2} y}{x+y}=\frac{x^{2}+x y-x y^{2}}{x+y} \\
& b=(1-x) y+\frac{x y^{2}}{x+y}=\frac{y^{2}+x y-x^{2} y}{x+y}
\end{aligned}
$$


under the constraints $(a, b) \in[0,1]^{2}$, and $0<a+b<1$. The explicit expression of $x$ and $y$ are difficult to obtain analytically (even with modern symbolic computing systems like Mathematica ${ }^{\mathrm{TM}}$, or Maple ${ }^{\mathrm{TM}}$ ) because one has a quartic equation to solve whose general analytical expression of its solutions is very complicate. Fortunately, the solutions can be easily calculated numerically by these computing systems, and even with Matlab ${ }^{\mathrm{TM}}$ system (thanks to the fsolve function) as soon as the numerical values are committed to $a$ and to $b$, and this is what we use in our simulations.

\section{Fast Fusion of dichotomous BBAs}

The main idea for making the fast fusion of dichotomous BBAs $m_{s}($.$) , for s=1,2, \ldots, S$ defined on the same FoD $\Theta$ is based on the three following main steps:

1) In the first step, one decomposes canonically each dichotomous BBA $m_{s}(\cdot)$ into its pro and contra evidences $m_{p, s}=\left(m_{p, s}(A), m_{p, s}(\bar{A}), m_{p, s}(A \cup \bar{A})\right)=\left(x_{s}, 0,1-\right.$ $\left.x_{s}\right)$ and $m_{c, s}=\left(m_{c, s}(A), m_{c, s}(\bar{A}), m_{c, s}(A \cup \bar{A})\right)=$ $\left(0, y_{s}, 1-y_{s}\right)$

2) In the second setp, one combines the pro-evidences $m_{p, s}$ for $s=1,2, \ldots, S$ altogether to get a global pro-evidence $m_{p}$, and in parallel one combines all the contra-evidences $m_{c, s}$ for $s=1,2, \ldots, S$ altogether to get a global contra-evidence $m_{c}$. The fusion step of pro and contra evidences is based on conjunctive rule of combination.

3) Once $m_{p}$ and $m_{c}$ are calculated, then one combines them with PCR5 fusion rule to get the final result.

Because the PCR5 rule of combination is not associative, the fusion of the canonical BBAs followed by their PCR5 fusion will not provide in general the same result as the direct fusion of the dichotomous BBAs altogether but only an approximate result, which is normal. However, this new fusion approach is interesting because the fusion of the pro-evidence $m_{p, s}$ (resp. contra-evidences $m_{c, s}$ ) is very simple because there is non conflict between $m_{p, s}$ (resp. between $m_{c, s}$ ), so that their fusion can be done quite easily and a large number of sources can be combined without a high computational burden. In fact, with this fusion approach, only one PCR5 fusion step of simple (combined) canonical BBAs is needed at the very end of the fusion process. In [5], we have proved with a Monte-Carlo simulation analysis that the approximation obtained by this new fusion method based on the fusion of pro-evidences and contra-evidences with respect to the direct fusion of the BBAs with PCR5 (or PCR6 when considering more than two sources to combine) is effective because the agreement between the decision taken from the direct fusion method, and the indirect (canonical decomposition based) method is very good. This new fusion method based on this canonical decomposition does not suffer of combinatorial complexity limitation which is of great interest in some applications because many (hundreds or even thousands) of dichotomous BBAs could be easily combined very quickly. Actually with this method what takes a bit time is only the canonical decomposition done by the numerical solver. Our analysis [5] has shown that complexity of this fast approach is quasi-linear with the number of sources to combine.

\section{THE BF-ICRA METHOD}

In [1], we did present an improved version of Atanassov's Inter-Criteria Analysis (ICrA) method [2]-[4] based on belief functions. This new method has been named BF-ICrA (Belief Function based Inter-Criteria Analysis) for short. It has already been applied to GPS surveying problems in [17]. We present briefly in this section the principles of BF-ICrA.

BF-ICrA starts with the construction of an $M \times N$ BBA matrix $\mathbf{M}=\left[m_{i j}(\cdot)\right]$ from the score matrix $\mathbf{S}=\left[S_{i j}\right]$. The BBA matrix $\mathbf{M}$ is obtained as follows - see [18] for details and justification.

$$
\begin{aligned}
m_{i j}\left(A_{i}\right) & =\operatorname{Bel}_{i j}\left(A_{i}\right) \\
m_{i j}\left(\bar{A}_{i}\right) & =\operatorname{Bel}_{i j}\left(\bar{A}_{i}\right)=1-P l_{i j}\left(A_{i}\right) \\
m_{i j}\left(A_{i} \cup \bar{A}_{i}\right) & =\operatorname{Pl}_{i j}\left(A_{i}\right)-\operatorname{Bel}_{i j}\left(A_{i}\right)
\end{aligned}
$$

where ${ }^{3}$

$$
\begin{aligned}
& \operatorname{Bel}_{i j}\left(A_{i}\right) \triangleq \operatorname{Sup}_{j}\left(A_{i}\right) / A_{\text {max }}^{j} \\
& \operatorname{Bel}_{i j}\left(\bar{A}_{i}\right) \triangleq \operatorname{Inf}_{j}\left(A_{i}\right) / A_{\min }^{j}
\end{aligned}
$$

with

$$
\begin{aligned}
& \operatorname{Sup}_{j}\left(A_{i}\right) \triangleq \sum_{k \in\{1, \ldots M\} \mid S_{k j} \leq S_{i j}}\left|S_{i j}-S_{k j}\right| \\
& \operatorname{Inf}_{j}\left(A_{i}\right) \triangleq-\sum_{k \in\{1, \ldots M\} \mid S_{k j} \geq S_{i j}}\left|S_{i j}-S_{k j}\right|
\end{aligned}
$$

and

$$
\begin{aligned}
& A_{\max }^{j} \triangleq \max _{i} \operatorname{Sup}_{j}\left(A_{i}\right) \\
& A_{\min }^{j} \triangleq \min _{i} \operatorname{In} f_{j}\left(A_{i}\right)
\end{aligned}
$$

For another criterion $C_{j^{\prime}}$ and the $j^{\prime}$-th column of the score matrix we will obtain another set of BBA values $m_{i j^{\prime}}(\cdot)$. Applying this method for each column of the score matrix we are able to compute the BBA matrix $\mathbf{M}=\left[m_{i j}(\cdot)\right]$ whose each component is in fact a triplet $\left(m_{i j}\left(A_{i}\right), m_{i j}\left(\bar{A}_{i}\right), m_{i j}\left(A_{i} \cup\right.\right.$ $\left.\left.\bar{A}_{i}\right)\right)$ of BBA values in $[0,1]$ such that $m_{i j}\left(A_{i}\right)+m_{i j}\left(\bar{A}_{i}\right)+$ $\left.m_{i j}\left(A_{i} \cup \bar{A}_{i}\right)\right)=1$ for all $i=1, \ldots, M$ and $j=1, \ldots, N$.

The next step of BF-ICrA approach is the construction of the $N \times N$ Inter-Criteria Matrix $\mathbf{K}=\left[K_{j j^{\prime}}\right]$ from $M \times N$ BBA matrix $\mathbf{M}=\left[m_{i j}(\cdot)\right]$ where elements $K_{j j^{\prime}}$ corresponds to the $\operatorname{BBA}\left(m_{j j^{\prime}}(\theta), m_{j j^{\prime}}(\bar{\theta}), m_{j j^{\prime}}(\theta \cup \bar{\theta})\right)$ about positive consonance $\theta$, negative consonance $\theta$ and uncertainty between criteria $C_{j}$ and $C_{j^{\prime}}$ respectively. The construction of the triplet $K_{j j^{\prime}}=\left(m_{j j^{\prime}}(\theta), m_{j j^{\prime}}(\bar{\theta}), m_{j j^{\prime}}(\theta \cup \bar{\theta})\right)$ is based on two steps:

- Step 1 (BBA construction): Getting $m_{j j^{\prime}}^{i}($.$) .$

For each alternative $A_{i}$ for $i=1, \ldots, M$, we first compute the $\mathrm{BBA}\left(m_{j j^{\prime}}^{i}(\theta), m_{j j^{\prime}}^{i}(\bar{\theta}), m_{j j^{\prime}}^{i}(\theta \cup\right.$

${ }^{3}$ assuming that $A_{\max }^{j} \neq 0$ and $A_{\min }^{j} \neq 0$. If $A_{\max }^{j}=0$ then $\operatorname{Bel}_{i j}\left(A_{i}\right)=0$, and if $A_{\min }^{j}=0$ then $P l_{i j}\left(A_{i}\right)=1$. 
$\bar{\theta})$ ) for any two criteria $j, j^{\prime} \in\{1,2, \ldots, N\}$. For this, we consider two sources of evidences (SoE) indexed by $j$ and $j^{\prime}$ providing the BBA $m_{i j}$ and $m_{i j^{\prime}}$ defined on the simple FoD $\left\{A_{i}, \bar{A}_{i}\right\}$ and denoted $m_{i j}=\left[m_{i j}\left(A_{i}\right), m_{i j}\left(\bar{A}_{i}\right), m_{i j}\left(A_{i} \cup \bar{A}_{i}\right)\right]$ and $m_{i j^{\prime}}=$ $\left[m_{i j^{\prime}}\left(A_{i}\right), m_{i j^{\prime}}\left(\bar{A}_{i}\right), m_{i j^{\prime}}\left(A_{i} \cup \bar{A}_{i}\right)\right]$. We also denote $\Theta=$ $\{\theta, \bar{\theta}\}$ the FoD about the relative state of the two SoE, where $\theta$ means that the two SoE agree, $\bar{\theta}$ means that they disagree and $\theta \cup \bar{\theta}$ means that we don't know. Hence, two SoE are in total agreement if both commit their maximum belief mass to the same element $A_{i}$ or to the same element $\bar{A}_{i}$. Similarly, two SoE are in total disagreement if each one commits its maximum mass of belief to one element and the other to its opposite, that is if one has $m_{i j}\left(A_{i}\right)=1$ and $m_{i j^{\prime}}\left(\bar{A}_{i}\right)=1$, or if $m_{i j}\left(\bar{A}_{i}\right)=1$ and $m_{i j^{\prime}}\left(A_{i}\right)=1$. Based on this very simple and natural principle, one can now compute the belief masses as follows:

$$
\begin{aligned}
& m_{j j^{\prime}}^{i}(\theta)=m_{i j}\left(A_{i}\right) m_{i j^{\prime}}\left(A_{i}\right)+m_{i j}(\bar{A}) m_{i j^{\prime}}(\bar{A}) \\
& m_{j j^{\prime}}^{i}(\bar{\theta})=m_{i j}\left(A_{i}\right) m_{i j^{\prime}}\left(\bar{A}_{i}\right)+m_{i j}\left(\bar{A}_{i}\right) m_{i j^{\prime}}\left(A_{i}\right) \\
& m_{j j^{\prime}}^{i}(\theta \cup \bar{\theta})=1-m_{j j^{\prime}}^{i}(\theta)-m_{j j^{\prime}}^{i}(\bar{\theta})
\end{aligned}
$$

$m_{j j^{\prime}}^{i}(\theta)$ represents the degree of agreement between the BBA $m_{i j}(\cdot)$ and $m_{i j^{\prime}}(\cdot)$ for the alternative $A_{i}, m_{j j^{\prime}}^{i}(\bar{\theta})$ represents the degree of disagreement of the two BBAs and $m_{j j^{\prime}}^{i}(\theta \cup \bar{\theta})$ the level of uncertainty (i.e. how much we don't know if they agree or disagree). By construction $m_{j j^{\prime}}^{i}(\cdot)=m_{j^{\prime} j}^{i}(\cdot), m_{j j^{\prime}}^{i}(\theta), m_{j j^{\prime}}^{i}(\bar{\theta}), m_{j j^{\prime}}^{i}(\theta \cup \bar{\theta}) \in[0,1]$ and $m_{j j^{\prime}}^{i}(\theta)+m_{j j^{\prime}}^{i}(\theta)+m_{j j^{\prime}}^{i}(\theta \cup \bar{\theta})=1$. This BBA modeling permits to build a set of $M$ symmetrical Inter-Criteria Belief Matrices (ICBM) $\mathbf{K}^{i}=\left[K_{j j^{\prime}}^{i}\right]$ of dimension $N \times N$ relative to each alternative $A_{i}$ whose components $K_{j j^{\prime}}^{i}$ correspond to the triplet of BBA values $m_{j j^{\prime}}^{i}=\left(m_{j j^{\prime}}^{i}(\theta), m_{j j^{\prime}}^{i}(\bar{\theta}), m_{j j^{\prime}}^{i}(\theta \cup \bar{\theta})\right)$ modeling the belief of agreement and of disagreement between $C_{j}$ and $C_{j^{\prime}}$ based on $A_{i}$.

- Step 2 (fusion): Getting $\mathbf{m}_{\mathbf{j} \mathbf{j}^{\prime}}($.$) .$

In this step, one needs to combine the BBAs $\mathbf{m}_{\mathbf{j} \mathbf{j}^{\prime}}^{\mathbf{i}}($. for $i=1, \ldots, M$ altogether to get the component $K_{j j^{\prime}}=\left(m_{j j^{\prime}}(\theta), m_{j j^{\prime}}(\bar{\theta}), m_{j j^{\prime}}(\theta \cup \bar{\theta})\right)$ of the InterCriteria Belief matrix ${ }^{4}(\mathrm{ICBM}) \mathbf{K}=\left[K_{j j^{\prime}}\right]$. For this and from the theoretical standpoint, we recommend to use the PCR6 fusion rule [12] (Vol. 3) because of known deficiencies of Dempster's rule.

Once the global Inter-Criteria Belief Matrix (ICBM) $\mathbf{K}=$ $\left[K_{j j^{\prime}}=\left(m_{j j^{\prime}}(\theta), m_{j j^{\prime}}(\bar{\theta}), m_{j j^{\prime}}(\theta \cup \bar{\theta})\right)\right]$ is calculated, we can identify the criteria that are in strong agreement, in strong disagreement, and those on which we are uncertain. For identifying the criteria that are in strong agreement, we evaluate the distance of each component of $K_{j j^{\prime}}$ with the BBA

\footnotetext{
${ }^{4}$ For presentation convenience, the ICBM $\mathbf{K}=\left[K_{j j^{\prime}}=\right.$ $\left.\left(m_{j j^{\prime}}(\theta), m_{j j^{\prime}}(\bar{\theta}), m_{j j^{\prime}}(\theta \cup \bar{\theta})\right)\right]$ is decomposed into three matrices $\mathbf{K}(\theta)=\left[K_{j j^{\prime}}^{\theta}=m_{j j^{\prime}}(\theta)\right], \mathbf{K}(\bar{\theta})=\left[K_{j j^{\prime}}^{\bar{\theta}}=m_{j j^{\prime}}(\bar{\theta})\right]$ and $\mathbf{K}(\theta \cup \bar{\theta})=\left[K_{j j^{\prime}}^{\theta \cup \bar{\theta}}=1-m_{j j^{\prime}}(\theta)-m_{j j^{\prime}}(\bar{\theta})\right]$.
}

representing the best agreement state and characterized by the specific $\mathrm{BBA}^{5} m_{T}(\theta)=1$. From a similar approach we can also identify, if we want, the criteria that are in very strong disagreement using the distance of $m_{j j^{\prime}}(\cdot)$ with respect to the BBA representing the best disagreement state characterized by the specific BBA $m_{F}(\bar{\theta})=1$. We use the belief interval distance $d_{B I}\left(m_{1}, m_{2}\right)$ presented in [19] for measuring the distance between the two BBAs.

\section{A. Fast BF-ICrA method}

The computational complexity of BF-ICrA is of course higher than the complexity of ICrA because it makes a more precise evaluation of local and global inter-criteria belief matrices with respect to inter-criteria matrices calculated by Atanassov's ICrA. The overall reduction of the computational burden of the original MCDM problem thanks to BF-ICrA depends highly on the problem under concern, the complexity and cost to evaluate each criteria involved in it, as well as the number of redundant criteria identified by BF-ICrA method.

The main drawback of BF-ICrA method is the PCR6 combination required in its step 2 for combining altogether the dichotomous BBAs $m_{j j^{\prime}}^{i}($.$) . Because of combinatorial$ complexity of PCR6 rule, it cannot work in reasonable computational time as soon as the number of sources to combine altogether is greater than 10 , which prevents its use for solving ICrA problems involving more than 10 alternatives (as in the examples 2 and 3 presented in section V). That is why it is necessary to adapt the original BF-ICrA method for working with a large number of alternatives and criteria. For this, we can in step 2 of BF-ICrA exploit the method for the fast fusion of dichotomous BBAs presented in section II-C. More precisely, each dichotomous BBA $m_{j j^{\prime}}^{i}($.$) will be$ canonically decomposed in its pro-evidence $m_{j j^{\prime}, p}^{i}($.$) and its$ contra-evidence $m_{j j^{\prime}, c}^{i}($.$) that will be combined separately to$ get the global pro-evidence $m_{j j^{\prime}, p}($.$) and the global contra-$ evidence $m_{j j^{\prime}, c}($.$) . Then, the BBAs m_{j j^{\prime}, p}($.$) and m_{j j^{\prime}, c}($. are combined with PCR5 rule to get the BBAs $m_{j j^{\prime}}($.$) and,$ finally, the global Inter-Criteria Belief Matrix $\mathbf{K}=\left[K_{j j^{\prime}}=\right.$ $\left.\left(m_{j j^{\prime}}(\theta), m_{j j^{\prime}}(\bar{\theta}), m_{j j^{\prime}}(\theta \cup \bar{\theta})\right)\right]$. The principle of this modified step 2 of BF-ICrA is summarized in the Figure 1 for convenience.

Another simpler fusion method to combine the dichotomous BBAs $m_{j j^{\prime}}^{i}$.) would just consist to average them. In section $\mathrm{V}$, we will show how these two methods behave in the examples chosen for the evaluation of MO-ACO Algorithm for optimal WSN deployment.

\section{Multi-Objective ACO Algorithm}

Recently Wireless Sensor Networks (WSNs) have attracted the attention of the research scientists community, conditioned by a set of challenges: theoretical and practical. WSNs consists of distributed sensor nodes and their main purpose is to monitor the real-time environmental status, based on gathering available sensor information, processing and transmitting the

\footnotetext{
${ }^{5}$ We use the index $T$ in the notation $m_{T}(\cdot)$ to refer that the agreement is true, and $F$ in $m_{F}(\cdot)$ to specify that the agreement is false.
} 


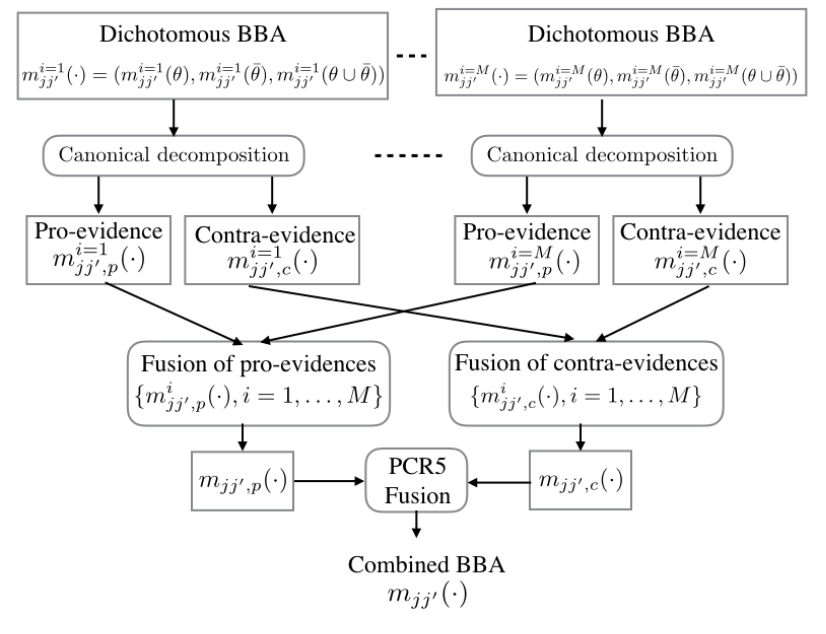

Figure 1. Principle of fast fusion of $m_{j j^{\prime}}^{i}($.$) of Step 2$ of BF-ICrA.

collected data to the specified remote base station. It is a promising technology that is used in a coverage of application requiring minimum human contribution, ranging from civil and military to healthcare and environmental monitoring. One of the key mission of WSN is the full surveillance of the monitoring region with a minimal number of sensors and minimized energy consumption of the network. The lifetime of the sensors is strongly related to the amount of the power loaded in the battery, that is why the control of the energy consumption of sensors is an important active research problem. The small energy storage capacity of sensor nodes intrudes the possibility to gather the information directly to the main base. Because of this they transfer their data to the so called High Energy Communication Node (HECN), which is able to collect the information from across the network and to transmit it to the base computer for processing. The sensors transmit their data to the HECN, either directly or via hops, using closest sensors as communication relays. The WSN can have large numbers of nodes and the problem can be very complex.

In order to solve successfully the key mission of WSNs, in [20], we did apply multi-objective Ant Colony Optimization (ACO) to solve this hard, from the computational point of view, telecommunication problem. The number of ants is one of the key algorithm parameters in the ACO and it is important to find the optimal number of ants needed to achieve good solutions with minimal computational resources. In [20], the optimal solution was obtained by applying the classical Atanassov's ICrA method. In the next section we will present the results obtained by the fast BF-ICrA approach and compare their results.

The problem of designing a WSN is multi-objective, with two objective functions: 1) one wants to minimize the energy consumption of the nodes in the network, and 2) one wants to minimize the number of nodes. The full coverage of the network and connectivity are considered as constraints. For solving this problem, we have proposed to use a $\mathrm{M}$ ulti-
Objective Ant Colony Optimization (MO-ACO) algorithm in [20] and we have studied the influence of the number of ants on the algorithm performance and quality of the achieved solutions. The computational resources, which the algorithm needs, are not negligible. The computational resources depends on the size of the solved problem and on the number of ants. The aim is to find a minimal number of ants which allow the algorithm to find good solution for WSN deployment.

The ACO algorithm uses a colony of artificial ants that behave as cooperating agents. With the help of the pheromone and the heuristic information they try to construct better solutions and to find the optimal ones. The pheromone corresponds to the global memory of the ants and the heuristic information is a some preliminary knowledge of the problem. The problem is represented by a graph and the solution is represented by a path in the graph or by tree in the graph. Ants start from random nodes and construct feasible solutions. When all ants construct their solution the pheromone is updated. The new, added, pheromone depends to the quality of the solution. The elements of the graph, which belong to better solutions will receive more pheromone and will be more desirable in the next iteration. In our implementation, we use the MAX-MIN Ant System (MMAS) which is one of the most successful ant approaches originally presented in [21]. In our case, the graph of the problem is represented by a square grid. The nodes of the graph are enumerated. The ants will deposit their pheromone on the nodes of the grid. We will deposit the sensors on the nodes of the grid too. The solution is represented by tree. An ant starts to create a solution starting from random node, which communicates with the HECN. Construction of the heuristic information is a crucial point in the ant algorithms. Our heuristic information represented by (21) is a product of three values.

$$
\eta_{i j}(t)=s_{i j} l_{i j}\left(1-b_{i j}\right)
$$

where $s_{i j}$ is the number of the new points (nodes of the graph) which the new sensor will cover, and which are not covered by other sensors, and

$$
l_{i j}= \begin{cases}1 & \text { if communication exists } \\ 0 & \text { if there is no communication. }\end{cases}
$$

and where $b_{i j}$ is the solution matrix. The matrix element $b_{i j}$ equals 1 when there is sensor on this position, otherwise $b_{i j}=0$. With $s_{i j}$, we try to increase the number of points covered by one sensor and thus to decrease the number of sensors we need. With $l_{i j}$, we guarantee that all sensors will be connected. With $b_{i j}$ we guarantee that maximum one sensor will be mapped on the same point. The search stops when transition probability $p_{i j}=0$ for all values of $i$ and $j$. It means that there are no more free positions, or that all area is fully covered. At the end of every iteration the quantity of the pheromone is updated according to the rule: $\tau_{i j} \leftarrow \rho \tau_{i j}+\Delta \tau_{i j}$, with the increment $\Delta \tau_{i j}=1 / F(k)$ if $(i, j)$ belongs to the non-dominated solution constructed by ant $k$, or $\Delta \tau_{i j}=0$ otherwise. The parameter $\rho$ is a pheromone 
decreasing parameter chosen in $[0,1]$. This parameter $\rho$ models evaporation in the nature and decreases the influence of old information on the search process. After that, we add the new pheromone, which is proportional to the value of the fitness function constructed as $F(k)=\frac{f_{1}(k)}{\max _{i}\left(f_{1}(i)\right)}+\frac{f_{2}(k)}{\max _{i}\left(f_{2}(i)\right)}$, where $f_{1}(k)$ is the number of sensors proposed by the $k$-th ant, and $f_{2}(k)$ is the energy of the solution of the $k$-th ant. These are also the objective functions of the WSN layout problem. We normalize the values of two objective functions with their maximal achieved values from the first iteration.

\section{APPLICATION OF THE FAST BF-ICRA METHOD}

In this section we present the results of the fast BFICrA method with the MO-ACO algorithm for WSN layout deployment. Fidanova and Roeva have developed a software, which realizes the MO-ACO algorithm. This software can solve the problem at any rectangular area, the communication and the coverage radius can be different and can have any positive value. We can have regions in the area. The program was written in $\mathrm{C}$ language, and the tests were run on computer with an Intel Pentium $2.8 \mathrm{GHz}$ processor. In their tests, they use an example where the area is square. The coverage and communication radii cover 30 points. The HECN is fixed in the centre of the area. In the sequel we consider three examples of areas with three sizes: $350 \times 350$ points, $500 \times 500$ points, and $700 \times 700$ points. The MO-ACO algorithm is based on 30 runs for each number of ants. We extract the Pareto front from the solutions of these 30 runs, and we show the achieved non dominated solutions (approximate Pareto fronts) for each case on which the BF-ICrA will be applied. The score matrices for each case is given in Tables I, II and III [20].

Table I

THE $6 \times 10$ SCORE MATRIX S FOR $350 \times 350$ CASE $($ EXAMPle 1$)$

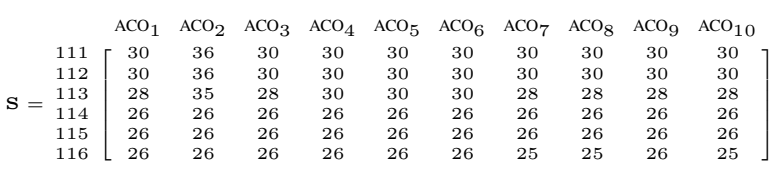

Each row of $\mathbf{S}$ corresponds to the number of sensors used in WSN to cover the area as indicated in the first column at the left side of the score matrix. Each column of $\mathbf{S}$ corresponds to $\mathrm{ACO}_{j}$ algorithm used with $j$ ants $(j=1,2, \ldots, 10)$. Each element $S_{i j}$ of $\mathbf{S}$ corresponds to the energy corresponding to this number of sensors and with the number of ants used for Multiple Objective ACO algorithm.

\section{Application of BF-ICrA in example $1(350 \times 350$ points $)$}

In this example, one sees from the score matrix of the Table I that $\mathrm{ACO}_{1}, \mathrm{ACO}_{3}$ and $\mathrm{ACO}_{9}$ algorithms perform equally for all alternatives (i.e. all rows) and they define a first group/cluster of methods providing exactly the same performances. Similarly, $\mathrm{ACO}_{4}, \mathrm{ACO}_{5}$ and $\mathrm{ACO}_{6}$ constitute a second group of algorithms. The third group is made of $\mathrm{ACO}_{7}$, $\mathrm{ACO}_{8}$ and $\mathrm{ACO}_{10}$ algorithms. It is worth noting that these
Table II

THE $22 \times 10$ SCORE MATRIX S FOR $500 \times 500$ CASE $($ EXAMPLE 2$)$.

\begin{tabular}{|c|c|c|c|c|c|c|c|c|c|c|}
\hline & $\mathrm{ACO}_{1}$ & $\mathrm{ACO}_{2}$ & $\mathrm{ACO}_{3}$ & $\mathrm{ACO}_{4}$ & $\mathrm{ACO}_{5}$ & $\mathrm{ACO}_{6}$ & $\mathrm{ACO}_{7}$ & $\mathrm{ACO}_{8}$ & $\mathrm{ACO}_{9}$ & $\mathrm{ACO}_{10}$ \\
\hline 223 & 90 & 96 & 90 & 90 & 89 & 81 & 90 & 90 & 90 & 90 \\
\hline 224 & 61 & 96 & 89 & 89 & 88 & 65 & 61 & 59 & 57 & 71 \\
\hline 225 & 61 & 96 & 74 & 58 & 60 & 58 & 57 & 58 & 57 & 57 \\
\hline 226 & 59 & 95 & 73 & 57 & 59 & 57 & 56 & 58 & 57 & 57 \\
\hline 227 & 60 & 57 & 57 & 57 & 57 & 56 & 56 & 57 & 57 & 57 \\
\hline 228 & 60 & 57 & 57 & 57 & 57 & 56 & 56 & 57 & 54 & 57 \\
\hline 229 & 58 & 57 & 57 & 55 & 57 & 56 & 56 & 56 & 54 & 56 \\
\hline 230 & 57 & 57 & 57 & 55 & 57 & 52 & 56 & 54 & 54 & 56 \\
\hline 231 & 57 & 55 & 57 & 55 & 55 & 52 & 56 & 54 & 54 & 56 \\
\hline 232 & 57 & 55 & 55 & 51 & 54 & 50 & 52 & 51 & 54 & 48 \\
\hline 233 & 57 & 55 & 55 & 51 & 54 & 50 & 51 & 51 & 54 & 48 \\
\hline & 57 & 55 & 55 & 51 & 53 & 50 & 51 & 48 & 53 & 48 \\
\hline 235 & 57 & 55 & 54 & 51 & 53 & 50 & 51 & 48 & 50 & 48 \\
\hline 236 & 57 & 55 & 54 & 51 & 53 & 50 & 51 & 48 & 50 & 48 \\
\hline 237 & 57 & 55 & 54 & 51 & 53 & 50 & 51 & 48 & 50 & 48 \\
\hline 238 & 57 & 55 & 53 & 51 & 53 & 50 & 51 & 48 & 50 & 48 \\
\hline 239 & 56 & 55 & 53 & 50 & 53 & 50 & 51 & 48 & 50 & 48 \\
\hline 240 & 53 & 53 & 53 & 50 & 53 & 50 & 51 & 48 & 50 & 48 \\
\hline 241 & 53 & 53 & 53 & 50 & 53 & 50 & 51 & 48 & 50 & 48 \\
\hline 242 & 53 & 53 & 53 & 50 & 53 & 50 & 51 & 48 & 50 & 48 \\
\hline 243 & 53 & 53 & 53 & 50 & 53 & 50 & 51 & 48 & 50 & 48 \\
\hline 244 & 53 & 53 & 53 & 50 & 52 & 50 & 51 & 48 & 50 & 48 \\
\hline
\end{tabular}

Table III

The $19 \times 10$ SCORE MATRIX S FOR $700 \times 700$ CASE (EXAMPle 3 ).

\begin{tabular}{|c|c|c|c|c|c|c|c|c|c|c|}
\hline & $\mathrm{ACO}_{1}$ & $\mathrm{ACO}_{2}$ & $\mathrm{ACO}_{3}$ & $\mathrm{ACO}_{4}$ & $\mathrm{ACO}_{5}$ & $\mathrm{ACO}_{6}$ & $\mathrm{ACO}_{7}$ & $\mathrm{ACO}_{8}$ & $\mathrm{ACO}_{9}$ & $\mathrm{ACO}_{10}$ \\
\hline 437 & 173 & 173 & 173 & 173 & 173 & 118 & 168 & 172 & 261 & 172 \\
\hline 438 & 173 & 173 & 173 & 173 & 173 & 118 & 112 & 117 & 260 & 172 \\
\hline 439 & 172 & 173 & 173 & 173 & 140 & 93 & 11 & 11 & 131 & 172 \\
\hline 440 & 172 & 17 & 17 & 17 & 11 & 9 & 11 & 1 & 1 & 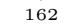 \\
\hline 441 & 172 & 173 & 173 & 122 & 111 & 93 & 110 & 114 & 111 & 110 \\
\hline 442 & 172 & 173 & 173 & 114 & 111 & 93 & 110 & 112 & 111 & 110 \\
\hline 443 & 172 & 150 & 123 & 114 & 111 & 93 & 110 & 112 & 111 & 110 \\
\hline 444 & 124 & 112 & 112 & 106 & 107 & 93 & 110 & 102 & 111 & 105 \\
\hline 445 & 117 & 112 & 112 & 106 & 107 & 93 & 110 & 102 & 108 & 105 \\
\hline$=446$ & 117 & 112 & 105 & 105 & 10 & 93 & 107 & 102 & 104 & 105 \\
\hline 447 & 117 & 112 & 105 & 105 & 105 & 93 & 105 & 102 & 102 & 105 \\
\hline 448 & 115 & 111 & 10 & 105 & 105 & 9 & 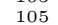 & 102 & 102 & 105 \\
\hline 449 & 11 & 1 & 10 & 10 & 10 & 9 & 102 & 99 & 16 & 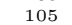 \\
\hline 450 & 11 & 1 & 1 & 10 & 10 & 9 & & 99 & 102 & 75 \\
\hline 451 & & 18 & 1 & 105 & 10 & 9 & 102 & 9 & 97 & 105 \\
\hline 452 & 113 & 109 & 105 & 105 & 105 & 93 & 99 & 99 & 97 & 104 \\
\hline 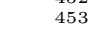 & 11 & $10 s$ & 1 & 105 & 105 & 93 & 99 & 99 & 9 & 104 \\
\hline 454 & 11 & 10 & 10 & 10 & 9 & 9 & 9 & 9 & 96 & 104 \\
\hline 455 & 106 & 106 & 105 & 105 & 96 & 93 & 96 & 96 & 96 & 97 \\
\hline
\end{tabular}

three groups $\left\{\mathrm{ACO}_{1}, \mathrm{ACO}_{3}, \mathrm{ACO}_{9}\right\},\left\{\mathrm{ACO}_{4}, \mathrm{ACO}_{5}, \mathrm{ACO}_{6}\right\}$, and $\left\{\mathrm{ACO}_{7}, \mathrm{ACO}_{8}, \mathrm{ACO}_{10}\right\}$ differ only very slightly, whereas the $\mathrm{ACO}_{2}$ algorithm (i.e the 2nd column of the score matrix S) differs a bit more from all the three aforementioned groups.

Example 1 with fast PCR6: If we apply the fast BF-ICrA method using approximate PCR6 fusion rule based on the canonical decomposition of the $M=6$ dichotomous BBAs $\left(m_{j j^{\prime}}^{i}(\theta), m_{j j^{\prime}}^{i}(\bar{\theta}), m_{j j^{\prime}}^{i}(\theta \cup \bar{\theta})\right)$, we get the matrix of mass of belief of agreement between criteria given in Table ${ }^{6}$ IV.

$$
\begin{gathered}
\text { Table IV } \\
\text { MATRIX } \mathbf{K}_{\approx P C R 6}(\theta) \text { FOR EXAMPLE } 1 .
\end{gathered}
$$

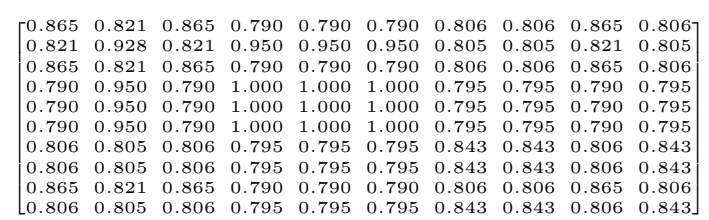

The matrix of distances to full agreement based on fast BFICrA method, denoted by $\mathbf{D}_{\approx P C R 6}(\theta)$, is given in Table $\mathrm{V}$.

${ }^{6}$ All the numerical values presented in the matrices have been truncated at their 3rd digit for typesetting convenience. 
Table V

MATRIX $\mathbf{D} \approx P C R 6(\theta)$ WITH FAST BF-ICRA FOR EXAMPLE 1 .

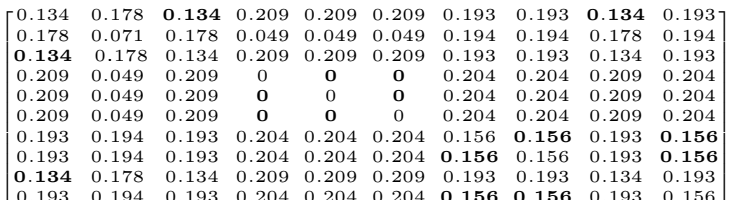

In examining the table $\mathrm{V}$, one sees that $A C O 1, A C O 3$ and $A C O 9$ are at a small distance 0.134 , with respect to other algorithms, so that they belong to the same group and behave similarly. Same remarks holds for the group $\left\{\mathrm{ACO}_{4}, \mathrm{ACO}_{5}, \mathrm{ACO}_{6}\right\}$ because its inter-distance is zero, and for the group $\left\{\mathrm{ACO}_{7}, \mathrm{ACO}_{8}, \mathrm{ACO}_{10}\right\}$ because its interdistance is 0.156. In a relative manner $\mathrm{ACO}_{2}$ appears closer to $\left\{\mathrm{ACO}_{4}, \mathrm{ACO}_{5}, \mathrm{ACO}_{6}\right\}$, than $\left\{\mathrm{ACO}_{1}, \mathrm{ACO}_{3}, \mathrm{ACO}_{9}\right\}$ or $\left\{\mathrm{ACO}_{7}, \mathrm{ACO}_{8}, \mathrm{ACO}_{10}\right\}$, which intuitively makes sense when comparing directly the columns of the matrix of Table I.

Example 1 with averaging fusion: The matrix of distances to full agreement based on BF-ICrA method using average fusion rule, denoted by $\mathbf{D}_{\text {Aver. }}(\theta)$, is given in Table VI.

Table VI

MATRIX D $\mathbf{D}_{\text {Aver. }}(\theta)$ WITh BF-ICRA USING AVERAGING RULE FOR EXAMPLE 1.

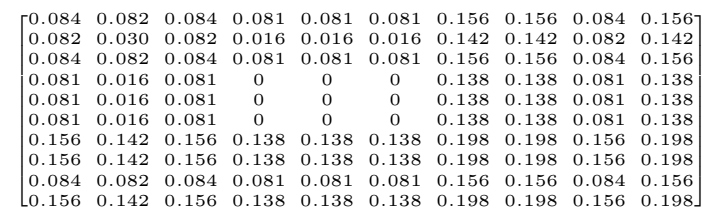

One sees that only the group $\left\{\mathrm{ACO}_{4}, \mathrm{ACO}_{5}, \mathrm{ACO}_{6}\right\}$ can be clearly identified based on the averaging fusion rule. The other groups $\mathrm{ACO}_{2}$ appears also close to $\left\{\mathrm{ACO}_{4}, \mathrm{ACO}_{5}, \mathrm{ACO}_{6}\right\}$. But $\mathrm{ACO}_{1}, \mathrm{ACO}_{3}$ and $\mathrm{ACO}_{9}$ are closer to $\left\{\mathrm{ACO}_{4}, \mathrm{ACO}_{5}, \mathrm{ACO}_{6}\right\}$ also than in-between. Same remarks holds for $\mathrm{ACO}_{7}, \mathrm{ACO}_{8}$, and $\mathrm{ACO}_{10}$. So one sees that the averaging fusion rule is not recommended for making the $\mathrm{BF}-\mathrm{ICrA}$ in this example.

Application of BF-ICrA in example $2(500 \times 500$ points $)$

Example 2 with fast PCR6: If we apply the fast BF-ICrA method using approximate PCR6 fusion rule based on the canonical decomposition of the $M=22$ dichotomous BBAs $\left(m_{j j^{\prime}}^{i}(\theta), m_{j j^{\prime}}^{i}(\bar{\theta}), m_{j j^{\prime}}^{i}(\theta \cup \bar{\theta})\right)$, we get the following matrix of distances to full agreement, denoted by $\mathbf{D}_{\approx P C R 6}(\theta)$, given in Table VII.

Based on these results, one sees that no clear group can be identified but we emphasize in boldface in Table VII the minimal value for each row of the distance matrix $\mathbf{D}_{\approx P C R 6}(\theta)$ (diagonal elements excluded). We see that $\mathrm{ACO}_{2}$ is at the farthest distance of $\mathrm{ACO}_{1}$ because $D_{12}(\theta)=0.376$, but in
Table VII

MATRIX $\mathbf{D} \approx P C R 6(\theta)$ WITH FAST BF-ICRA FOR EXAMPLE 2.

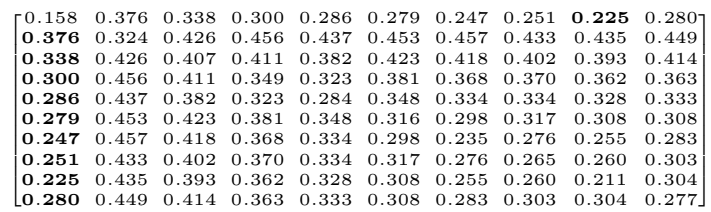

the mean time $\mathrm{ACO}_{2}$ is at closest distance to $\mathrm{ACO}_{1}$ because $D_{2 j}(\theta)>0.376$ (for $j>2$ ) as shown in second line of Table VII. So we can conclude that $\mathrm{ACO}_{2}$ is not close to any other algorithm in fact. If we choose a ad-hoc distance threshold, say for instance 0.28 , then we can identify the group $\left\{\mathrm{ACO}_{1}, \mathrm{ACO}_{7}, \mathrm{ACO}_{8}, \mathrm{ACO}_{9}\right\}$.

Example 2 with averaging fusion: The matrix of distances to full agreement based on BF-ICrA method using average fusion rule, denoted by $\mathbf{D}_{\text {Aver. }}(\theta)$, is given in Table VIII.

Table VIII

MATRIX $\mathbf{D}_{\text {Aver. }}(\theta)$ WITH BF-ICrA USING AVERAGING RULE FOR EXAMPLE 2 .

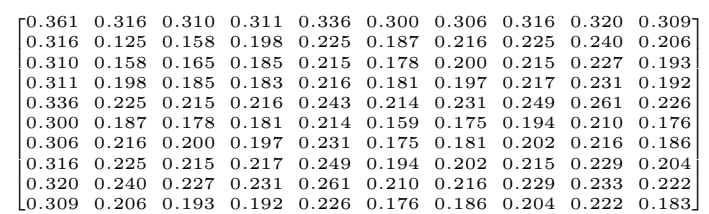

Based on the average fusion rule there is no clear clustering of algorithms. However based on shortest interdistance we could make the following distinct pairwise groupings $\left\{\mathrm{ACO}_{2}, \mathrm{ACO}_{3}\right\},\left\{\mathrm{ACO}_{6}, \mathrm{ACO}_{7}\right\}, \quad\left\{\mathrm{ACO}_{4}, \mathrm{ACO}_{10}\right\}$, $\left\{\mathrm{ACO}_{8}, \mathrm{ACO}_{9}\right\}$ and $\left\{\mathrm{ACO}_{1}, \mathrm{ACO}_{5}\right\}$ if necessary, but remember that average fusion rule cannot provide the best result as shown in Example 1.

\section{Application of BF-ICrA in example $3(700 \times 700$ points $)$}

Example 3 with fast PCR6: If we apply the fast BF-ICrA method using approximate PCR6 fusion rule based on the canonical decomposition of the $M=19$ dichotomous BBAs $\left(m_{j j^{\prime}}^{i}(\theta), m_{j j^{\prime}}^{i}(\bar{\theta}), m_{j j^{\prime}}^{i}(\theta \cup \bar{\theta})\right)$, we get the matrix of distances to full agreement, denoted by $\mathbf{D}_{\approx P C R 6}(\theta)$, given in Table IX.

Table IX

MATRIX $\mathbf{D} \approx P C R 6(\theta)$ WITH FAST BF-ICRA FOR EXAMPLE 3.

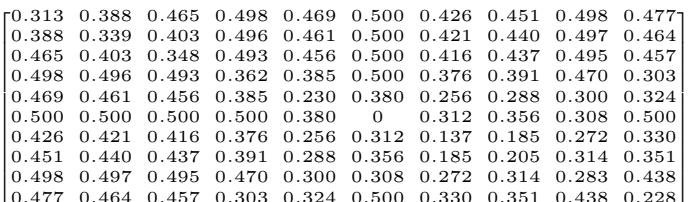

We observe that the average distance between ACO algorithms is much higher than in Tables V and VII of examples 
1 and 2. This shows clearly the difficulty to precisely identify the clusters of similar algorithms because only few ACO algorithms perform actually very well for this third example. Eventually, and based on shortest inter-distance we could make the first pairwise group $\left\{\mathrm{ACO}_{7}, \mathrm{ACO}_{8}\right\}$ because $D_{78}(\theta)=$ 0.185 is the minimal inter-distance we have between the ACO algorithms. Once the rows and columns of Table IX corresponding to $\mathrm{ACO}_{7}$ and $\mathrm{ACO}_{8}$ are eliminated, then the second best group will be $\left\{\mathrm{ACO}_{5}, \mathrm{ACO}_{9}\right\}$ because $D_{59}(\theta)=0.300$. Similarly, we will get the group $\left\{\mathrm{ACO}_{4}, \mathrm{ACO}_{10}\right\}$ because $D_{4,10}(\theta)=0.303$, and then the group $\left\{\mathrm{ACO}_{1}, \mathrm{ACO}_{2}\right\}$ because $D_{12}(\theta)=0.388$. Finally we could also cluster $\mathrm{ACO}_{3}$ with $\mathrm{ACO}_{6}$ because $D_{36}(\theta)=0.500$, although this distance of agreement is quite large to be considered as a trustable cluster.

Example 3 with averaging fusion: The matrix of distances to full agreement based on BF-ICrA method using average fusion rule, denoted by $\mathbf{D}_{\text {Aver. }}(\theta)$, is given in Table $X$.

Table X

MAtrix $\mathbf{D}_{\text {Aver. }}(\theta)$ WITH BF-ICrA USING AVERAGING RULE FOR EXAMPLE 3.

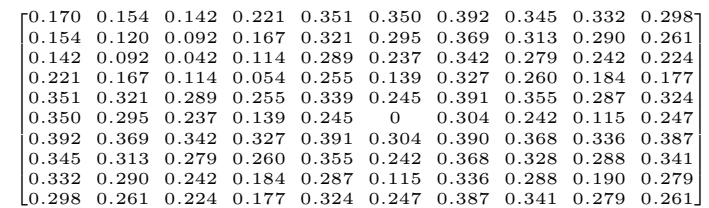

Surprisingly, the use of averaging rule provides in this example lower distance values on average with respect to values given in Table IX. However no clear clustering of algorithms can be made because only few ACO algorithms perform actually very well for this third example. If we adopt the pairwise strategy to cluster algorithms, we will obtain now as first group $\left\{\mathrm{ACO}_{2}, \mathrm{ACO}_{3}\right\}$ because $D_{23}(\theta)=0.092$, as second group $\left\{\mathrm{ACO}_{6}, \mathrm{ACO}_{9}\right\}$ because $D_{69}(\theta)=0.115$, as third group $\left\{\mathrm{ACO}_{4}, \mathrm{ACO}_{10}\right\}$ because $D_{4,10}(\theta)=0.177$, as fourth group $\left\{\mathrm{ACO}_{1}, \mathrm{ACO}_{8}\right\}$ because $D_{18}(\theta)=0.345$, and finally we could also cluster $\mathrm{ACO}_{5}$ with $\mathrm{ACO}_{7}$ because $D_{57}(\theta)=0.391$. one sees that there is no strong correlation between results obtained from BF-ICrA based on fast PCR6 and those based on averaging rule, which is not surprising because the rules are totally different. Nevertheless the group $\left\{\mathrm{ACO}_{4}, \mathrm{ACO}_{10}\right\}$ is agreed by both methods here.

\section{CONCLUSIONS}

The fast Belief Function based Inter-Criteria Analysis method, using the canonical decomposition of basic belief assignments defined on a dichotomous frame of discernment was applied, tested and analysed in this paper. This new method was applied for evaluating the Multiple-Objective Ant Colony Optimization (MO-ACO) algorithm for Wireless Sensor Networks (WSN) deployment. Based on the BF-ICrA outcomes it was shown a very high correlation with fast
PCR6 rule for the $\mathrm{ACO}_{1}, \mathrm{ACO}_{3}$ and $\mathrm{ACO}_{9}$ group, for the $\mathrm{ACO}_{4}, \mathrm{ACO}_{5}$ and $\mathrm{ACO}_{6}$ group, and for the $\mathrm{ACO}_{7}, \mathrm{ACO}_{8}$ and $\mathrm{ACO}_{10}$ group of algorithms in example 1 (case of size $350 \times 350$ ) as intuitively expected. This is because the considered ACO algorithms can solve the problem with good solution quality in example 1. These high correlations were not observed in the other two cases for example 2 (case of size $500 \times 500$ ) and 3 (case of size $700 \times 700$ ) because only few ACO algorithms perform actually very well for these examples. So, if we considered results in case of larger problem sizes, the BF-ICrA results show that the number of ants has the significant influence on the obtained results, as already pointed out in [20].

\section{ACKNOWLEDGMENTS}

This work is partially supported by the grant No BG05M20P001-1.001-0003, financed by the Science and Education for Smart Growth Operational Program (2014-2020) and co-financed by the European Union through the European structural and Investment funds and the Bulgarian scientific fund by the grant DN 12/5.

\section{REFERENCES}

[1] J. Dezert, A. Tchamova, D. Han, J.-M. Tacnet, Simplification of multicriteria decision-making using inter-criteria analysis and belief functions, in Proc. of Fusion 2019 Int. Conf. on Information Fusion, Ottawa, Canada, July 2-5, 2019.

[2] K. Atanassov, D. Mavrov, V. Atanassova, Intercriteria decision making: a new approach for multicriteria decision making, based on index matrices and intuitionistic fuzzy sets. Issues IFSs GNs 11, pp. 1-8, 2014.

[3] K. Atanassov, V. Atanassova, G. Gluhchev, InterCriteria Analysis: Ideas and problems, Notes on IFS, Vol. 21, No. 1, pp. 81-88, 2015.

[4] K. Atanassov et al., An approach to a constructive simplification of multiagent multicriteria decision making problems via intercriteria analysis, C.R. de l'Acad. Bulgare des Sci., Vol. 70, No. 8, 2017.

[5] J. Dezert, F. Smarandache, A. Tchamova, D. Han, Fast Fusion of Basic Belief Assignments Defined on a Dichotomous Frame of Discernment, In Proc. of Fusion 2020 (Online) conference, Pretoria, South Africa, July 2020.

[6] J. Dezert, F. Smarandache, Canonical Decomposition of Dichotomous Basic Belief Assignment, International Journal of Intelligent Systems, pp. 1-21, 2020.

[7] G. Shafer, A Mathematical Theory of Evidence, Princeton Univ. Press, 1976.

[8] J. Dezert, P. Wang, A. Tchamova, On the validity of Dempster-Shafer theory, Proc. of Fusion 2012, Singapore, July 9-12, 2012.

[9] A. Tchamova, J. Dezert, On the Behavior of Dempster's Rule of Combination and the Foundations of Dempster-Shafer Theory, IEEE IS2012, Sofia, Bulgaria, Sept. 6-8, 2012.

[10] J. Dezert, A. Tchamova, On the validity of Dempster's fusion rule and its interpretation as a generalization of Bayesian fusion rule, Int. J. of Intelligent Syst., Vol. 29, Issue 3, pages 223-252, March 2014.

[11] F. Smarandache, J. Dezert, On the consistency of PCR6 with the averaging rule and its application to probability estimation, Proc. of Fusion 2013, Istanbul, Turkey, July 2013.

[12] F. Smarandache, J. Dezert (Editors), Advances and applications of DSmT for information fusion, American Research Press, Vol. 1-4, 2004-2015, http://www.onera.fr/staff/jean-dezert?page $=2$

[13] F. Smarandache, J. Dezert, J.-M. Tacnet, Fusion of sources of evidence with different importances and reliabilities, in Proceedings of Fusion 2010 conference, Edinburgh, UK, July 2010.

[14] https://bfasociety.org/

[15] R. Yager, On the Dempster-Shafer framework and new combination rules, Information Sciences, Vol. 41, pp. 93-138, 1987.

[16] D. Dubois, H. Prade, Representation and combination of uncertainty with belief functions and possibility measures, Comput. Intell., 4, 1988. 
[17] S. Fidanova, J. Dezert, A. Tchamova, Inter-criteria analysis based on belief functions for GPS surveying problems, in Proc. of IEEE Int. Symp. on INnovations in Intelligent SysTems and Applications (INISTA 2019), Sofia, Bulgaria, July 3-5, 2019.

[18] J. Dezert, D.Han, H. Yin, A New Belief Function Based Approach for Multi-Criteria Decision-Making Support, Proc. of Fusion 2016 Conf.

[19] D. Han, J. Dezert, Y. Yang, New Distance Measures of Evidence based on Belief Intervals, Proc. of Belief 2014, Oxford, UK, Sept. 2014.

[20] S. Fidanova, O. Roeva, Multi-objective ACO Algorithm for WSN Layout: InterCriteria Analysis, in Large-Scale Scientific Computing, Springer, 2020.

[21] M. Dorigo, T. Stutzle, Ant Colony Optimization, MIT Press, Cambridge, 2004. 